\title{
ARTICLE
}

\section{Development of intense gamma-ray source monitoring system in water for radiation safety}

\author{
Hiroyuki Miyamaru*, Takao Kojima, Kiwamu Omura and Ryoichi Taniguchi \\ Radiation Research Center, Osaka Prefecture University, 2-1 Gakuen-cho, Nakaku, Sakai Osaka,599-8531, Japan
}

\begin{abstract}
A gamma-ray dose measurement system with a small-sized cesium iodide scintillator and a long-optical-fiber combination is developed to monitor an intense cobalt-60 gamma-ray radiation source in a deep-water pool, for radiation safety. The compact sensor consists of cesium iodide doped with thallium scintillator and a light focusing lens component. The luminescence of the scintillator, as the gamma-ray response, is collected using the lens and introduced into the $10 \mathrm{~m}$-long optical fiber. Finally, the light output from the sensor is input to a photomultiplier and converted to an output voltage corresponding to the luminescence intensity. A $940 \mathrm{TBq}$ cobalt-60 gamma-ray source is placed at the pool bottom and used as a target for the monitoring experiment. The dose rate to water is measured with two sensors located at different depth positions. By moving the sensors horizontally, the dose rates at several positions within the pool are measured and compared. The distance to the source can be estimated from the dose rate measured at the sensor position. Observations of the dose rate changes by moving both the sensors vertically against the pool bottom indicate that the motion of the source in an upper direction can be detected using two sensors located at different depths.
\end{abstract}

Keywords: radiation safety; cobalt-60 gamma-ray radiation source; gamma-ray dose measurement; cesium iodide scintillator; long optical fiber

\section{Introduction}

An intense cobalt-60 $\left({ }^{60} \mathrm{Co}\right)$ gamma-irradiation facility at Osaka Prefecture University (OPU) has been developed for more than 50 years [1]. This facility owns several gamma-irradiation sources, which are being used extensively for various purposes such as academic research, education, and technical tests. The total activity of the source is approximately $2.4 \mathrm{PBq}$ and the irradiation dose rate reaches a maximum of approximately $50 \mathrm{kGy} / \mathrm{h}$. Of late, we are involved in a human resource development project related to the decommissioning of a nuclear plant in our facility and are offering the use of the intense sources for research. Although these sources are stored and handled safely in a water pool, higher radiation protection safety is now required for handling such intense sources. Hence, we are currently developing a monitoring system that enables the online measurement of the radiation dose at several points in water. This system is designed to monitor the position of the source and its movement by transfer operation in the pool. It enables the continuous monitoring of the source position in water and is expected to contribute in increasing the radiation safety. The characteristics of the developed system are

\footnotetext{
*Corresponding author. Email: miyamaru@riast.osakafu-u.ac.jp
}

discussed below.

\section{Equipment}

Figure 1 displays the image of the developed sensors. The compact gamma-ray sensor consists of a small-sized scintillator and a light focusing lens component with a fiber optic connector (FC) (Edmund Optics Inc., 64777). Cesium iodide, which is rectangular in shape with $1 \mathrm{~cm}$ square and $3 \mathrm{~cm}$ in length, doped with thallium scintillator, is placed within a plastic case. One-side of the scintillator is in close contact with the lens, for efficiently guiding the scintillation to an optical fiber. The scintillator component is wrapped with white-thread seal tape for light reflection and is fully covered with a rubber tube for waterproofing and light shielding. The luminescence of the scintillator, as a gamma-ray response, is collected with the lens and introduced into a $10 \mathrm{~m}$-long optical fiber connected to the sensor and finally, to a photomultiplier. The optical fiber has an acrylic polymer polymethyl-methacrylate (PMMA) core with a diameter of $2 \mathrm{~mm}$ and is covered with a black polyethylene jacket. Using the optical fiber transmission technique, electrical devices such as photoelectric sensors and power supplies can be handled in air. This contributes not only to the protection of the electronic equipment but also to a significant decrease in the 


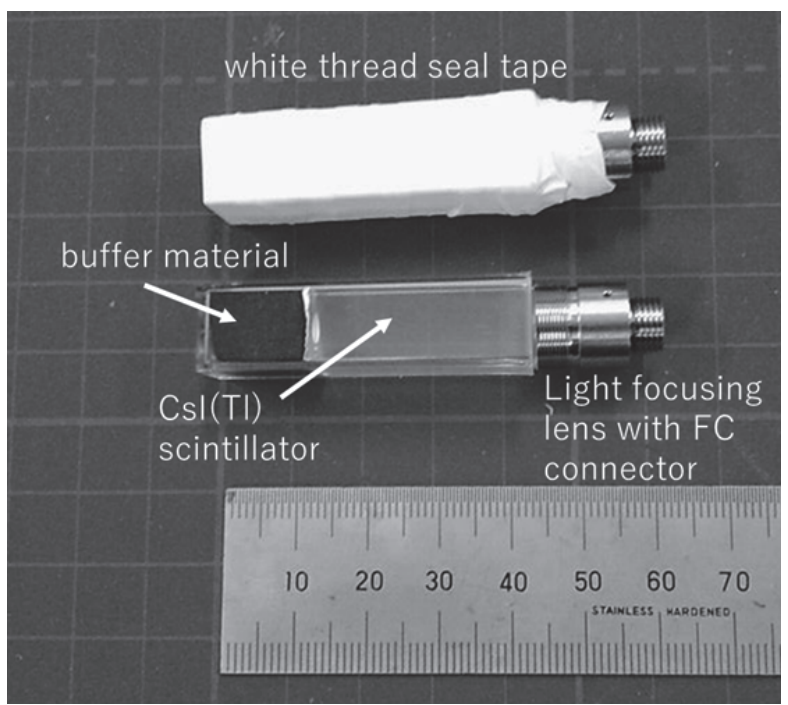

Figure 1. Photo of the sensor assembly.

background noise by the intense radiation

field. The light output from the sensor is finally input to a photomultiplier (Hamamatsu Photonics k.k. H10722) and converted into a DC voltage output proportional to the quantity of light. The output is measured with a digital multimeter (Yokogawa, TY720). A 0.3-cc volume ion chamber (PTW, Type31013) and a dosemeter (PTW, UNIDOS E) are used, along with two sensors, for exposure measurement. All the measured data with exposure rate units $(\mathrm{C} / \mathrm{kg} \mathrm{sec})$ are converted into absorbed dose rate to water $(\mathrm{mGy} / \mathrm{h})$, using an f-factor of $37.6 \mathrm{~Gy} \mathrm{~kg} / \mathrm{C}$ for water [2], with the assumption of a charged particle equilibrium condition. The f-factor is known as the Roentgen-to-Rad conversion factor. We have employed the absorbed dose rate to water as an index, instead of the exposure, in the following discussion.

The experiment was performed at the radiation research center in OPU. Figure 2 illustrates the experimental setup in a water pool, 3-m wide, 5-m long, and 4.7-m deep. A $940 \mathrm{TBq}{ }^{60} \mathrm{Co}$ gamma-ray source was placed at the pool bottom as a target for monitoring. Four ${ }^{60} \mathrm{Co}$ rods were stored in a cylindrical stainless container, $10-\mathrm{cm}$ in diameter and $50-\mathrm{cm}$ in height.

Currently, our monitoring system involves two sensors located at different depths in the pool. The sensors are used to monitor the radiation dose at the sensor position in water; the position of the radiation source and its variation are estimated based on the changes in the dose rate. Changes in the source position towards the upper direction (water surface) should be monitored carefully for radiation safety because such a situation may occur accidentally by inappropriate operation. Therefore, it is critical to detect the motion in the upper direction rapidly; thus, the two sensors are arranged in series along the water depth direction. In the experiment, the sensors were fixed to a $5 \mathrm{~m}$-long polyvinyl chloride (PVC) pipe, which was sunk vertically in the water surface, as shown in Figure 2. The sensors were located at $50 \mathrm{~cm}$ and at $150 \mathrm{~cm}$, respectively, from the pool bottom. Instead of moving the source, the sensor pipe was moved horizontally, maintaining the direction perpendicular to the water surface. The outputs of both the sensors and the exposure rate measured with the ion chamber positioned at the same depth as that of the lower sensor were measured at several different horizontal positions in the pool. This moving operation is intended to simulate a situation in which fixed sensors monitor the source position and its horizontal change. The horizontal distance between the source and sensor pipe was measured by trilateration with two laser distance meters (BOSCH GLM50C) placed at fixed positions on the pool edge. Distance measurements to a pipe appearing on the water surface can be performed with the distance meter that has an uncertainty of $1.5 \mathrm{~mm}$; however, the

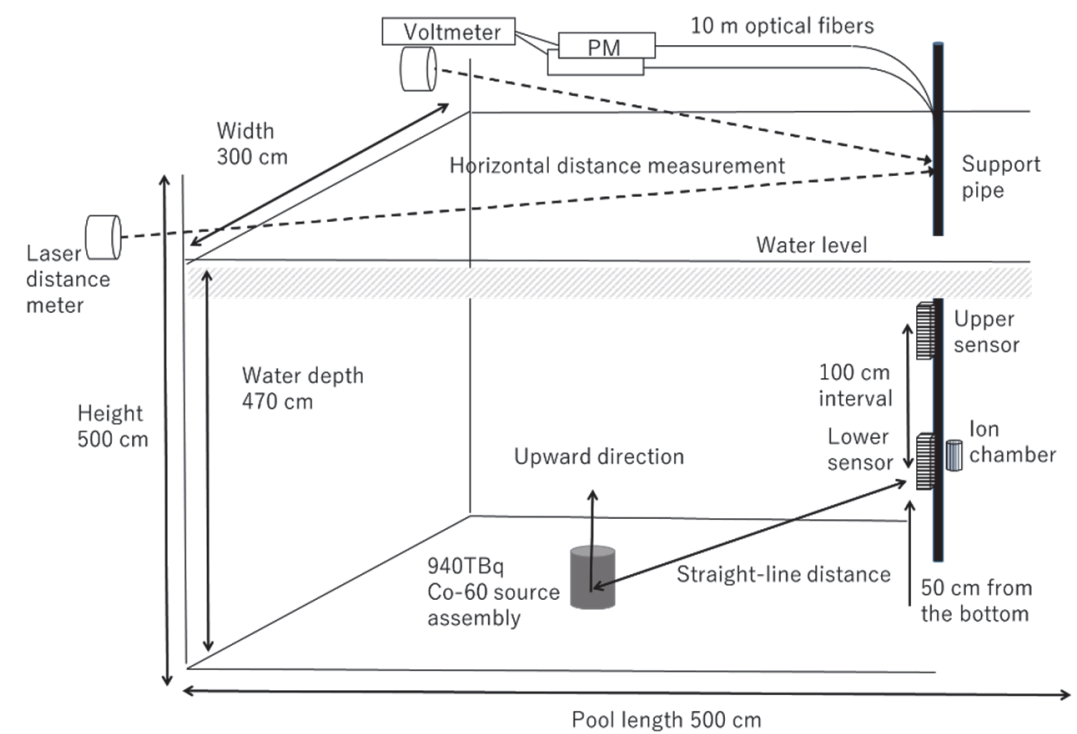

Figure 2. Experim ental setup for dose rate measurement in water. 
sensor position in deep water has an uncertainty of a few centimeters because of slight deflections in the long pipe; this uncertainty is taken account in the measured distance value. The straight-line distance between the source and sensor was finally calculated from both the horizontal and vertical values. For evaluating the distance between the source and sensor, the center of the source volume was considered to be the starting point of the distance measurement. Lifting and lowering operations of the sensor pipe were also performed to measure the vertical (upward direction) changes in the source position.

\section{Results and discussion}

The sensors and the ion chamber were irradiated with the same dose rate as in the pool and the output of each sensor was calibrated as a function of the dose rate. The sensor response showed a linear relationship over a wide dose range, as shown in Figure 3. In our experiment, the same control voltage value for gain adjustment was applied to both the photomultipliers. The gain difference is possibly owing to the individual differences in the photomultipliers. The sensitivity of our system depends upon various factors such as the luminescence collection efficiency, scintillator volume, and the transmission efficiency of the optical fiber. These factors are not adjustable, but the sensitivity can be finally controlled by adjusting the gain of the photomultiplier, after complete system assembly. In this study, the maximum measurable dose rate was set to be approximately 40 $\mathrm{mGy} / \mathrm{h}$. In a high-dose condition, the optical fiber and focusing lens may emit a luminescence by themselves, adding to the response output. Hence, the output voltage of the sensor without the scintillator was experimentally examined at a dose irradiation of $30 \mathrm{mGy} / \mathrm{h}$. The measured value was of the order of a few millivolts, considerably larger than the background output corresponding to the photomultiplier dark current; however, it corresponds to less than $1 \%$ of the output voltage from the sensor equipped with the scintillator. Consequently, the light emissions from the lens and fiber were found to be sufficiently small. The small-volume ion chamber, used in this study, was not suitable for calibration at a lower dose rate region because of the dose sensitivity. The uncertainty in the dose rate was evaluated from the uncertainty of the measured exposure rate of the ion chamber and its value was determined to be $0.6 \mathrm{mGy} / \mathrm{h}$.

When a spherically symmetric dose distribution in a water volume is assumed to be formed by the radiation source at the pool bottom, the dose rate at the sensor position is determined using only the straight-line distance between the source and the sensor, and its change can be approximately expressed as an exponential function of the straight-line distance because of attenuation by water.

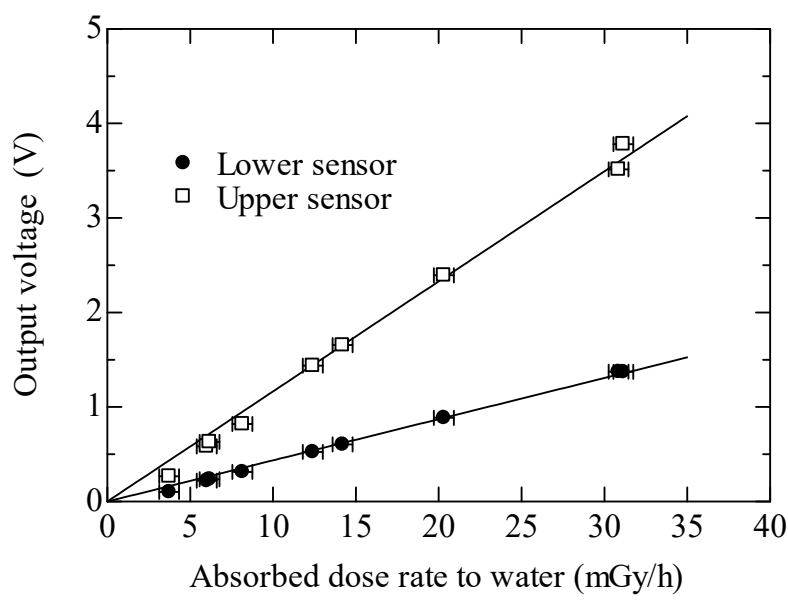

Figure 3. Relationship between the absorbed dose rate and the output voltage of the sensor.

The measured values of both the sensors were gathered and summarized as a function of the straight-line distance, in Figure 4. In order to compare the measured value, a Monte Carlo simulation of the gamma-ray transport in water was performed using the particle and heavy-ion transport code system (PHITS Ver. 2.88) [3], under a calculation condition that includes the pool structure, such as the $3 \mathrm{~mm}$-thick stainless-steel plate at the bottom, the concrete bottom wall under the plate, and the stainless-steel source holder. A heat tally was used to evaluate the absorbed dose to water and the photon history number was 625 million, in this simulation.

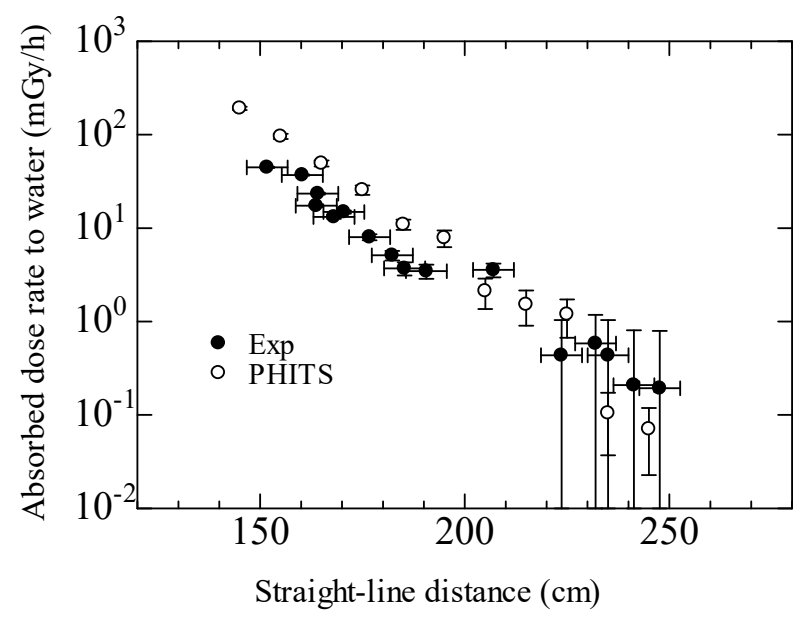

Figure 4. Absorbed dose rate as a function of the straight-line distance between the radiation source and sensor.

The simulation result is also plotted as open circles in the figure. The absorbed dose rate reduces as an exponential function of the distance and the measured values show a similar tendency. The dose rate calculated with the PHITS is slightly higher than the measured value. Because of insufficient statistics, the uncertainty of the calculated values becomes considerably large at far distance, indicating that changes in the distance cause significant changes in the dose rate, at the sensor 
position. A position change of $50 \mathrm{~cm}$ along the straight-line direction results in a magnitude change of approximately an order in the dose rate. When the source moves in the straight-line direction, its movement can be detected easily. In this case, the dose rate change is considerably large and a change in the source position by a few tens of centimeters can be detected with high sensitivity. On the other hand, it can be expected that the sensitivity will be poor in the case of position changes perpendicular to the straight-line direction. Assuming that the initial straight-line distance is $180 \mathrm{~cm}, 50 \mathrm{~cm}$ position change of the source to the perpendicular direction causes only $7 \mathrm{~cm}$ increase of the straight-line distance; this may be insufficient to identify its change. From this result we determined that the distance to the source can be estimated from the dose rate at the sensor position and that the sensitivity for detecting the position change had a dependency on the direction of the source movement.

The responses of the two sensors were examined, while moving the pipe vertically from the bottom of the pool. The upper sensor locates $100 \mathrm{~cm}$ higher than the lower one in depth, as described before. This experiment is intended to detect a situation in which the radiation source rises slightly from the bottom to the water surface direction, accidentally. The horizontal distance was kept to be $155 \mathrm{~cm}$ in this experiment. The dose rate of each sensor is plotted as a function of the distance from the pool bottom, as in Figure 5. For a horizontal change in the source position, the sensitivity of the upper sensor is not sufficient owing to the distance. For a vertical change, the dose rate of both the sensors vary with respect to the change in depth, as indicated in the figure. Significant dose rate increases in the upper sensor enable us to detect the motion of the source in an upward direction clearly.

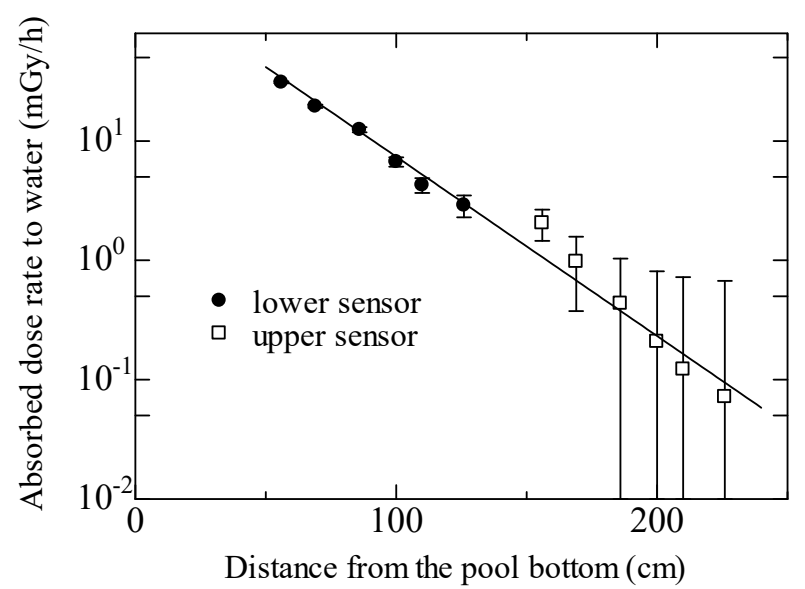

Figure 5. Absorbed dose rate as a function of the distance from the pool bottom, for each sensor.

\section{Conclusion}

In this study, a scintillator sensor with a long optical fiber transmission was developed for monitoring an intense radiation source in water. The dose rate at the sensor position changes markedly with changes in the straight-line distance from the source; this characteristic can be used for detecting the position changes of the intense radiation source in water. Although our current study uses only two sensors, increasing the number of sensors can provide more information on the source position and motion. When the sensor is located close to the source, the sensitivity improves but the dose rate may exceed the limit easily, even for slight changes in the source position. On the contrary, the sensitivity will be insufficient for monitoring at distant positions. Hence, it is crucial to determine the appropriate sensitivity requirement and the acceptable dose range of the sensor. Our monitoring system can be applied to a wider dose range by the several adjustments, for example, selection of the scintillator volume, filtering of an intense scintillation and the gain adjustment of the photomultiplier. The photon counting method, whose application for lower dose rate monitoring is currently under investigation, will be effective for monitoring from a distance. Further study is in progress to establish a monitoring system for radiation safety.

\section{References}

[1] J. Furuta, E. Hiraoka, S. Okamoto, M. Fujishiro, T. Kanazawa, T. Ohnishi, Y. Tsujii and T. Azuma, On the new gamma-irradiation facility, Annual Report of the Radiation Center of Osaka Prefecture 10 (1970), pp. 65-70.

[2] F.M. Khan and J.P. Gibbons, Khan's The Physics of Radiation Therapy fifth edition, Wolters Kluwer, (2014), ISBN 978-1-4511-8245-3.

[3] T. Sato, K. Niita, N. Matsuda, S. Hashimoto, Y. Iwamoto, S. Noda, T. Ogawa, H. Iwase, H. Nakashima, T. Fukahori, K. Okumura, T. Kai, S. Chiba, T. Furuta and L. Sihver, Particle and heavy ion transport code system PHITS, version 2.52, $\mathrm{J}$. Nucl. Sci. Technol. 50:9 (2013), pp. 913-923. 